\title{
Theory of Transport of Conteiners in Pipelines
}

\author{
František Klimenda ${ }^{1, *}$, Josef Soukup ${ }^{1}$, and Lenka Rychlíková ${ }^{1}$ \\ ${ }^{1}$ University of J. E. Purkyně, Faculty of Mechanical Engineering, Institute of Machinery and \\ Energetics, Ústí nad Labem, Czech Republic
}

\begin{abstract}
For transporting materials pneumatic transport in containers is also used. Calculation of this type of transport is quite complicated, especially because of non-stationary and non-isothermal conditions in the pipes. Because of low speeds of the train motion in the pipes, it is possible to consider the phenomenon in the pipes isothermal. The simplified calculation of this type of transport for one-dimensional flow in the pipes is indicated in the article.
\end{abstract}

\section{Introduction}

Pneumatic transport of materials in containers is not very widespread. It is most used only in Russia and the states of the former Soviet Union (especially in the Caucasus, where the terrain is inaccessible). Although it is used on such a small scale, it is an interesting and energy-saving mode of transport. This mode of transport in the large-diameter pipeline has been developed to transport raw materials in inaccessible terrains or in challenging climatic conditions. It is possible to imagine its use, for example, for transporting goods in today's long-range gas pipelines. The transport system is presented by pipe tube in which the containers on the wheels are moved in the driving gas (air) stream supplied by the compressor stations. The pressure gradient on the containers is generated by automatically. The energy of the air stream is transferred to the containers.

At low container speeds the pressure gradient is small, for the movement of containers there is no need for great force [1, 4]. By measuring it was found that due to the energy losses and the costs of the piping system, the optimum container speed is between 15 and $30 \mathrm{~km} \cdot \mathrm{h}^{-1}$. At higher speeds, although energy losses are lower, it is necessary to build a massive transport pipeline with guide rails, which significantly increases costs.

Typically, these systems operate at a compression pressure of $100-200 \mathrm{kPa}$ (overpressure). Containers run on wheels at the bottom of the pipeline. The guide wagons also run along the top of the pipeline. The assembly of the entire container train consists of end guides and sealing wagons. Containers with cargo are loaded between these wagons.

The source of air for the drive of the containers are turbochargers, vacuum pumps or ventilators. Depending on the air source and the length of the route, we recognize the systems

- magistral - for long-distance traffic, they are always overpressure,

- industrial - overpressure,

\footnotetext{
${ }^{*}$ Corresponding author: frantisek.klimenda@,ujep.cz
} 


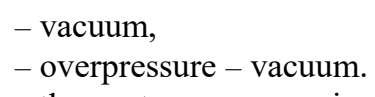

Depending on the route, we recognize the systems branched, unbranched, straight and circular. The containers are loaded and unloaded at stations. The stations are done as end (end of route) or as travel (between end stations). The whole system may be one-way, ie. all trains have one loading and one unloading station or multi-directional - can be interpreted on the route or trains run to the branching.

The diameter of the pipeline is dependent on the amount of conveyance, the size of the container, the specific profile of the track and its required throughput. Large pipe diameters are used. More than 1 million tons of material per year are used with two or more piping automated systems.

The container train assembly consists of freight containers, sealing wagons and coupling devices (see Figure 1).

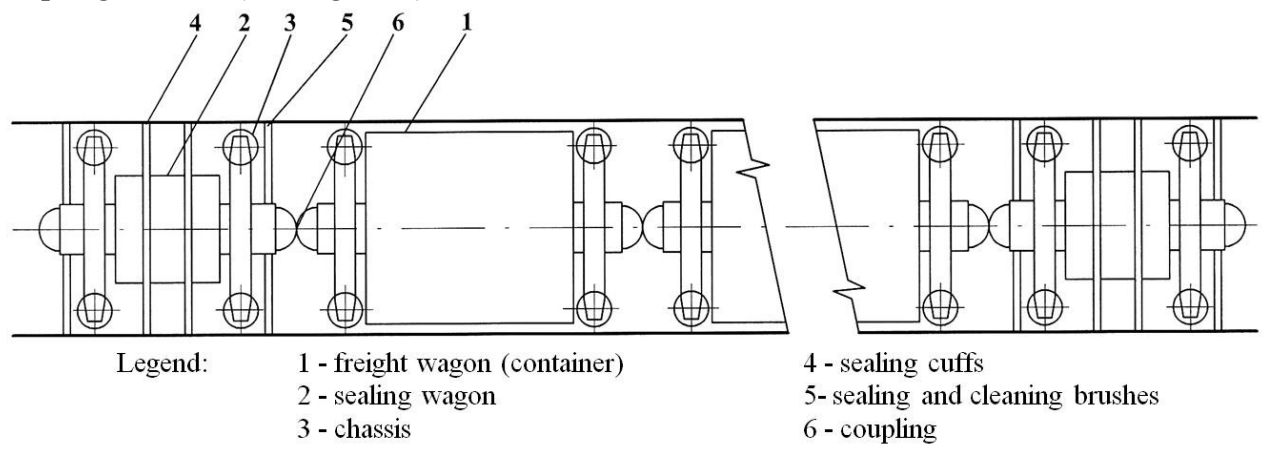

Fig. 1. train assembly - schema [4].

\section{Calculation}

The movement of the container assembly in the pipeline is described by the usual differential equations II. order. The force acting on the set is a pressure gradient on pneumatic wagons (sealing wagons located at the beginning and end of the container train). The pressure $p$ and the amount of air $Q_{v}$ characterize the air source. For simplicity, the flow of propellant gas in the pipeline is considered to be one-dimensional [2].

The basic equations describing the one-dimensional flow of gas in a pipeline for continuous (homogeneous) environments are based on the Law of conserving mass (1), momentum (2) and energy (3). The system of differential equations of the one-dimensional flow of gas in the pipeline is characterized by the flow in the axis. We draw from the gas dynamics equation in which all functions represent the values in the center of the cross section and depend only on the position $x$ and time $t$.

Basic equation [1]

$$
\begin{gathered}
\frac{\mathrm{d}}{\mathrm{d} t} \int_{V} \rho \cdot \mathrm{d} \tau=0 \\
\frac{\mathrm{d}}{\mathrm{d} t} \int_{V} \rho \cdot w \cdot \mathrm{d} \tau=\oint_{\Omega} \mathbf{P}_{n} \mathrm{~d} \sigma+\int_{V} \rho \cdot \mathbf{F} . \mathrm{d} \tau \\
\frac{\mathrm{d}}{\mathrm{d} t} \int_{V} \rho\left(u+\frac{w^{2}}{2}\right) \mathrm{d} \tau=\oint_{\Omega} \mathbf{P}_{n} w \mathrm{~d} \sigma+\int_{V} \rho \cdot \mathbf{F} . w \cdot \mathrm{d} \tau+\oint_{\Omega} q_{n} \mathrm{~d} \sigma
\end{gathered}
$$


where $\mathbf{P}_{n}$ - surface pressure with normal $n, \mathbf{F}$ - mass forces, $u$ - internal energy, $w-$ velocity of the gas (medium), $q_{n}$ - Heat flow in the normal direction, $\rho$ - gas mass, $V-$ volume, $\Omega$ - surface bounding volume.

It is not appropriate to describe the whole theoretical process. After editing and derivation we get the equation of the heat input

$$
\rho \frac{\mathrm{d} u}{\mathrm{~d} t}=\frac{p}{\rho} \frac{\mathrm{d} \rho}{\mathrm{d} t}+\mathbf{T} \nabla \mathbf{w}+\operatorname{div} \mathbf{q}
$$

where $\mathbf{T}$ - tensor of tangent pressures (forces), $p$ - pressure.

The calculation involves consideration of changes in enthalpy, internal energy and gas pressure, as well as heat input. For the one-dimensional flow in the pipeline (which we use for these cases in the technological calculations) we modify the equations so that all the variables depend only on the position $(x)$ and the time $(t)$, ie to the shape

$$
A(x, t)=\langle A(x, y, z, t)\rangle=\frac{4}{\pi d^{2}} \int_{S} A(x, y, z, t) d \sigma
$$

where $S$ - sectional area of pipeline, $d$-pipe diameter, $A$ - function.

After editing the equation, they will have a shape

$$
\begin{aligned}
& \frac{\partial \rho}{\partial t}+\frac{\partial Q}{\partial x}=0 \quad Q=\rho \cdot w \\
& \frac{\partial Q}{\partial t}+\frac{\partial}{\partial x}\left(p+\beta \cdot \rho \cdot w^{2}\right)=\frac{4}{d} \tau+\rho \cdot F_{x} \\
& \rho \frac{\mathrm{d} u}{\mathrm{~d} t}-\frac{p}{\rho} \frac{\mathrm{d} \rho}{\mathrm{d} t}=\langle\mathbf{T} \nabla \mathbf{w}\rangle+\frac{4}{d} q_{n}+\frac{\partial q_{x}}{\partial x}
\end{aligned}
$$

where $Q(x, t)$ - the amount of gas, $w$ - medium gas velocity in pipeline, $p$-gas pressure in pipeline, $\tau$ - the mean tensile stress (gas) on the inner surface of the pipe, $\beta$-coefficient of unequal distribution of gas velocity $\mathrm{w}$ in the cross section of the pipeline, for turbulent flow $\beta \approx 1, q_{n}, q_{x}$ - radial and axial heat flux.

Further modifications and simplifications, we obtain 6 equations determining the relationship between $\rho, p, w, Q, T$ and $u$

$$
\begin{aligned}
& \frac{\partial \rho}{\partial t}+\frac{\partial Q}{\partial x}=0 \quad \frac{\partial Q}{\partial t}+\frac{\partial p}{\partial x}=-\lambda \frac{1}{d} \frac{\rho \cdot w \cdot|w|}{2} \\
& \rho \frac{\mathrm{d} u}{\mathrm{~d} t}-\frac{p}{\rho} \frac{\mathrm{d} Q}{\mathrm{~d} t}=\lambda \frac{1}{d} \frac{w^{3}}{2}+\frac{4 \cdot q_{n}}{d} \\
& u=c \cdot v \cdot T+\text { konst. } \quad p=\rho \cdot R \cdot T \quad Q=\rho \cdot w
\end{aligned}
$$

After adjusting and fitting from these equations, we determine the amount of air required to drive the containers in the pipeline. The air is fed behind the container train, the air in front of it, until the next full-pass shutter is led by a passage through the blowers to the next section. For a coherent (homogeneous) environment, we come out of the law on the conservation of matter, energy and movement.

In the solution, we assume that

- the weight, pressure, internal energy and gas temperature change slightly over the pipe cross-section

- the gas velocity on the inner surface is equal to zero

- gas compression is much greater than the flexibility of the pipe walls.

Then we get

$$
\frac{\partial Q}{\partial t}+\frac{\partial Q}{\partial x}=0
$$


after editing

$$
\frac{\partial Q}{\partial t}+\frac{\partial}{\partial x}\left(p+\beta \cdot \rho \cdot w^{2}\right)=\frac{4}{d} \tau+\rho \cdot F x
$$

Further simplification can be achieved by hydraulic approach (by conversion to hydraulic flow conditions). When introducing simplification - the surface tension on the inner surface of the pipeline is associated with the average flow parameters.

According to Darcy

$$
\frac{4}{d} \tau=-\lambda \frac{1}{d} \frac{\rho \cdot w(w)}{2}
$$

where $\lambda$ - dimensionless coefficient of hydraulic resistance.

This simplification ceases to apply in high speed processes - gas velocity compared to the gas velocity (Mach number)

$$
M=\frac{w}{c}<<1
$$

This simplification does not consider the convective transfer of motion in the equation of motion, ie the variable $\rho w^{2}$ compared to the pressure fluctuations. The fluctuation of pressure according to Zhukovsky is [3]

$$
\partial_{p}=c . \partial Q
$$

and fluctuations $\partial\left(\beta \rho w^{2}\right) \approx \beta . w . \partial Q$

The ratio of fluctuations of the given variables

$$
\frac{\partial\left(\beta \cdot \rho \cdot w^{2}\right)}{\partial_{p}} \approx \beta \frac{w}{e}
$$

The heat flow along the $x$-axis is much larger than the $y$-axis flow.

From the equations of the non-stationary isothermal movement of the gas in the pipeline after the modifications we get

$$
\frac{\partial p}{\partial t}+c^{2} \frac{\partial Q}{\partial x}=0
$$

In the quasi-isothermal movement of gas in the pipeline, the equation system is nonlinear $(c=$ const.). Linearization of equations allows to find an analytical solution for many tasks of non-stationary motion using rows or operating methods.

The presence of container assemblies in the gas flow is the task of moving the containers in the non-linear pneutage in the case where the gasodynamic equations undergo linearization.

In addition, the complexity of the track profile, the non-linearity of boundary conditions, and the need to monitor multiple container assemblies in the pipeline while moving, leads to solving tasks using numerical methods using computer technology. The description of the solution (ie the integration of equations) will not be addressed in this paper.

For the non-isothermal flow of the equation we modify the shape

$$
\begin{gathered}
\frac{\partial p}{\partial t}-\frac{p}{T} \frac{\partial T}{\partial t}+\frac{c^{2}}{\kappa} \frac{\partial Q}{\partial x}=0 \\
\frac{\partial Q}{\partial t}+\frac{\partial p}{\partial x}=\varphi(p, Q, T)
\end{gathered}
$$




$$
\begin{aligned}
& c_{p} \rho \frac{\partial T}{\partial t}+c_{p} \cdot Q \frac{\partial T}{\partial x}-\frac{\partial p}{\partial t}=\varepsilon(T) \\
& p=\rho \cdot R \cdot T \quad c^{2}=\kappa \cdot R \cdot T \\
& \varphi(p, Q, T)=-\frac{\lambda c^{2}(T) Q / Q}{2 \cdot \kappa \cdot d \cdot p}
\end{aligned}
$$

Sometimes it is necessary (especially for long routes) to solve systems with high density sets. The calculation is complex, it is solved with Dirac functions, double integers, etc. When designing a container pneumatic transport system, we perform basic technological calculations.

System throughput

$$
Q_{i} \leq Q_{i \max } \quad i=(m, V, s, t)
$$

quantity of material transported per year $Q_{r}$ we need

$$
m=\frac{Q_{r}}{q} \text { containers }
$$

where $q$ - the capacity of one container.

We have $n$ containers in one set, then we have to go through the year

$$
v=\frac{m}{n} \quad \text { sets }
$$

Then the interval of the movement of the container assemblies

$$
\tau=\frac{e}{v}
$$

The movement of the container sets is characterized by the fact that in the closed part of the length $l_{i}$ (between the passage closures) there is always only one container set. The throughput, ie, the duration of the stay one of the container set when moving back and forth is given by the relationship

where

$$
\tau_{\min }=\tau_{p}+\tau_{r}+\frac{2 L}{v_{\max }}
$$

$\tau_{p}, \tau_{r}$ - time of unloading and time of loading, $L$ - pipe length, $v_{\max }$ - maximum mean speed.

In case of two-pipe systems, the duration of the kit is one half in the pipeline and therefore $\tau_{\min }$ is also half.

\section{Conclusion}

The diameter of the conveying duct depends on the amount transported, the container size, the specific profile of the track and the required throughput. Large pipe diameters are used.

Several features have been realized, all of which are used to transport building materials, even in hard-to-reach terrain. Typically, the pipeline diameter is between 1.020 $1.220 \mathrm{~m}$ with a pitch up to $3^{\circ}$. The most important is the TDO transport system in St. Petersburg. It works with parameters: pipe diameter $1.220 \mathrm{~m}$, climb max. $2^{\circ}$, full train train weight is $20 \mathrm{t}$, empty $10.9 \mathrm{t}$, transmission capacity is $125000 \mathrm{t} /$ year [4].

For horizontal transport there is a pressure drop of about $15 \mathrm{kPa}$, at a climb of about 45 $\mathrm{kPa}$. The pressure gradient forms sealing wagons. 
The research work is supported by the UJEP-SGS-2017-48-003-3, Czech Republic.

\section{References}

1. J. Soukup, Pneumatická doprava odpadi̊, Habilitační práce, 2004

2. J. Janalík, Potrubni hydraulická a pneumatická doprava, Ostrava, VŠB v Ostrvě, 1984, 209 s.

3. J. Urban, Pneumatická doprava, SNTL Praha, 1964

4. J. Soukup, Nové způsoby odstraňováni odpadi̊ (pneumatická doprava), Výzkumný ústav výstavby a architektury, Praha, 1990 